\title{
Clinically isolated syndrome and multiple sclerosis in children: a single center study
}

\author{
Gonca Bektaş ${ }^{1 \oplus}$, Melis Ulak Özkan ${ }^{2 \oplus}$, Edibe Pembegül Yıldız ${ }^{2 \oplus,}$ \\ Tuğçe Aksu Uzunhan ${ }^{2 \oplus}$, Serra Sencer ${ }^{3 \oplus}$, Nur Aydınlı ${ }^{2 \oplus}$, Mine Çalışkan² ${ }^{2 \oplus}$ \\ Meral Özmen ${ }^{2 \odot}$ \\ ${ }^{1}$ Department of Pediatric Neurology, Bakırköy Dr. Sadi Konuk Research and Training Hospital, Istanbul; Departments of ${ }^{2}$ Pediatric \\ Neurology and ${ }^{3}$ Neuroradiology, İstanbul University Istanbul Faculty of Medicine, İstanbul, Turkey.
}

\begin{abstract}
Background and objectives. This study was conducted to determine the differences in clinical and radiological features at the first demyelinating event in children with clinically isolated syndrome (CIS) and multiple sclerosis (MS).

Methods. This was a single center retrospective cohort study of the children with CIS followed-up at Istanbul University Faculty of Medicine, Department of Pediatric Neurology, between 2010 and 2018. Children with CIS who were assessed at 3, 6, 12 and 24 months following their first identified demyelinating event were included. Demographic data, mode of presentation and the presence of the oligoclonal band in the cerebrospinal fluid (CSF) were abstracted from the medical records. Magnetic resonance imaging of the brain and spinal cord was analyzed for the location, number, size and gadolinium enhancement of the lesions.
\end{abstract}

Results. A total of 51 patients' data was assessed, 38 patients at a mean age of 12.3 years were enrolled in the study. Twenty-seven children (71\%) evolved into clinically definite MS after a mean follow-up of 11 months. Older age at first demyelinating event and the presence of the oligoclonal band in CSF were tended to be more common in patients with MS than patients with CIS ( $\mathrm{p}<0.05)$. The increased number of T2-hyperintense lesion and the presence of the lesion in periventricular, infratentorial and corpus callosum were associated with a tendency for development of MS ( $\mathrm{p}<0.05)$.

Conclusion. Older age at first demyelinating event, the presence of the oligoclonal band in CSF, the number and localization of T2-hyperintense lesion were associated with a tendency for development of MS.

Key words: child, clinically isolated syndrome, multiple sclerosis.

Multiple sclerosis (MS), an acquired inflammatory demyelinating disease of the central nervous system (CNS), has its onset during childhood in 3-5\% of MS patients. ${ }^{1}$ The treatment approach focuses on prevention of demyelinating event recurrence, which may lead to severe physical disability. The International Pediatric Multiple Sclerosis Study Group (IPMSSG) has suggested initiating immunomodulatory therapy promptly in

$\triangle$ Gonca Bektaş

goncabektas@gmail.com

Received 28th February 2019, revised 16th May 2019, accepted 21st June 2019. children diagnosed with MS to prevent disease progression. $^{2}$

Clinically isolated syndrome (CIS), an inflammatory demyelinating disease of the CNS, refers to the first clinical event of the CNS leading to symptoms and signs for at least 24 h. ${ }^{3}$ The studies on adult patients with CIS stated the positive prognostic effect of early initiation of immunomodulatory therapy prior to MS diagnosis., ${ }^{4,5}$ However, immunomodulatory treatment has not been suggested in children with CIS. Treatment approaches may improve by identifying children with a higher risk for developing MS. 
In this study, we aimed to determine the differences in clinical and radiological features at the first demyelinating event in children with CIS and MS and to identify children with CIS at higher risk of developing MS.

\section{Material and Methods}

This was a single center retrospective cohort study of the children with CIS followed-up at the Istanbul University Faculty of Medicine, Department of Pediatric Neurology, in Turkey between January 1, 2010, and January 31, 2018.

\section{Participants}

Children with CIS according to the revised International Pediatric Multiple Sclerosis Study Group criteria who had a clinical and radiological assessment at 3, 6, 12 and 24 months following their first identified demyelinating event were included. ${ }^{6,7}$ CIS was diagnosed according to following criteria; (1) clinical CNS event with presumed inflammatory demyelinating cause which could be monofocal or polyfocal, (2) the first CNS demyelinating disease, (3) no encephalopathy, (4) baseline MRI which has not fulfilled the requirements to diagnose MS. ${ }^{7}$ The exclusion criteria was the presence of clinical and radiological features resembling neuromyelitis optica disorder at the first demyelinating event (e. g. bilateral optic neuritis, longitudinally extensive transverse myelitis). Children were diagnosed with clinically definite MS in case of 2 or more nonencephalopathic clinical events with presumed inflammatory cause occurring in a 30 day interval in presence of MRI evidence of dissemination in space, or 1 nonencephalopathic clinical event with MRI evidence of dissemination in space and in which follow-up MRI emerges at least one new lesion. ${ }^{6,7}$ None of the patients with CIS received immunomodulatory drugs prior to MS diagnosis.

The study was reviewed and approved by the Institutional Ethics Committee at the Istanbul University Faculty of Medicine (Date:
08.11.2017, No: 13). All participants and/or their legal representatives provided a written informed consent and assent.

\section{Data collection}

Demographic and clinical data including age, sex, mode of presentation, the season of the first event, and cerebrospinal fluid (CSF) oligoclonal band status were abstracted from the medical records. The season of the first event was classified as winter (Dec 1-Feb 28), spring (March 1-May 30), summer (June 1-Aug 30), or autumn (Sept 1- Nov 31). All CSF specimens were analyzed at Istanbul University Faculty of Medicine by isoelectric focusing.

The required magnetic resonance imaging (MRI) protocol was 1.5 Tesla brain MRI which consisted of T2-weighted/FLAIR, pre- and post- gadolinium T1-weighted sequences in the sagittal and axial planes. Spinal cord MRI scans were in the axial and sagittal planes on Tl-weighted and T2-weighted imaging. MRI of the brain and spinal cord was analyzed by an experienced neuroradiologist blinded to the clinical features for the location, number, size and gadolinium enhancement of the lesions. ${ }^{6}$ White matter hyperintensities greater than 5 $\mathrm{mm}$ in diameter on T2-weighted or FLAIR MRI images were counted. Lesions greater than $2 \mathrm{~cm}$ in diameter were classified as giant lesions, and lesions involving more than two adjacent gyri were considered as confluent lesions.

\section{Statistical analysis}

Quantitative variables were described as mean and standard deviation. Frequencies and percentages were calculated. Qualitative clinical and MRI characteristics were compared using Fischer's exact test, and quantitative clinical and MRI characteristics were compared using the Student's t-test. $p<0.05$ was accepted as statistically significant. All statistical analyses were performed using SPSS Statistics for Windows version 21.0 (IBM Corp., Armonk, NY, USA). 


\section{Results}

A total of 51 patients' data was assessed for eligibility, 38 patients at a mean age of 12.3 years (range 3 to 17, standard deviation [SD] 3.4) were enrolled in the study. Twenty-seven children $(71 \%)$ evolved into clinically definite MS after a mean follow-up of 11 months (range 1 to 61 months, SD 13.3). The earliest MS development occurred in a boy aged 12-yearsold who presented with diplopia and horizontal right conjugate gaze palsy. The baseline axial FLAIR MRI showed the hyperintense lesions in the periventricular white matter and brainstem and diffusion-weighted and apparent diffusion coefficient imaging showed high signal intensity resembling stroke in the brain stem. Therefore, we performed a follow-up MRI. He was diagnosed with MS by emerging new T2hyperintense lesions on brain MRI 1 month after the first event, type II pattern was observed by isoelectric focusing of the CSF (Fig. 1).

The remaining 11 children (29\%) showed disease progression neither clinically nor radiologically during a total of 7 years of followup (mean 4.9 years, SD 1.8). Demographic and clinical features of children with CIS and MS are presented in Table I.

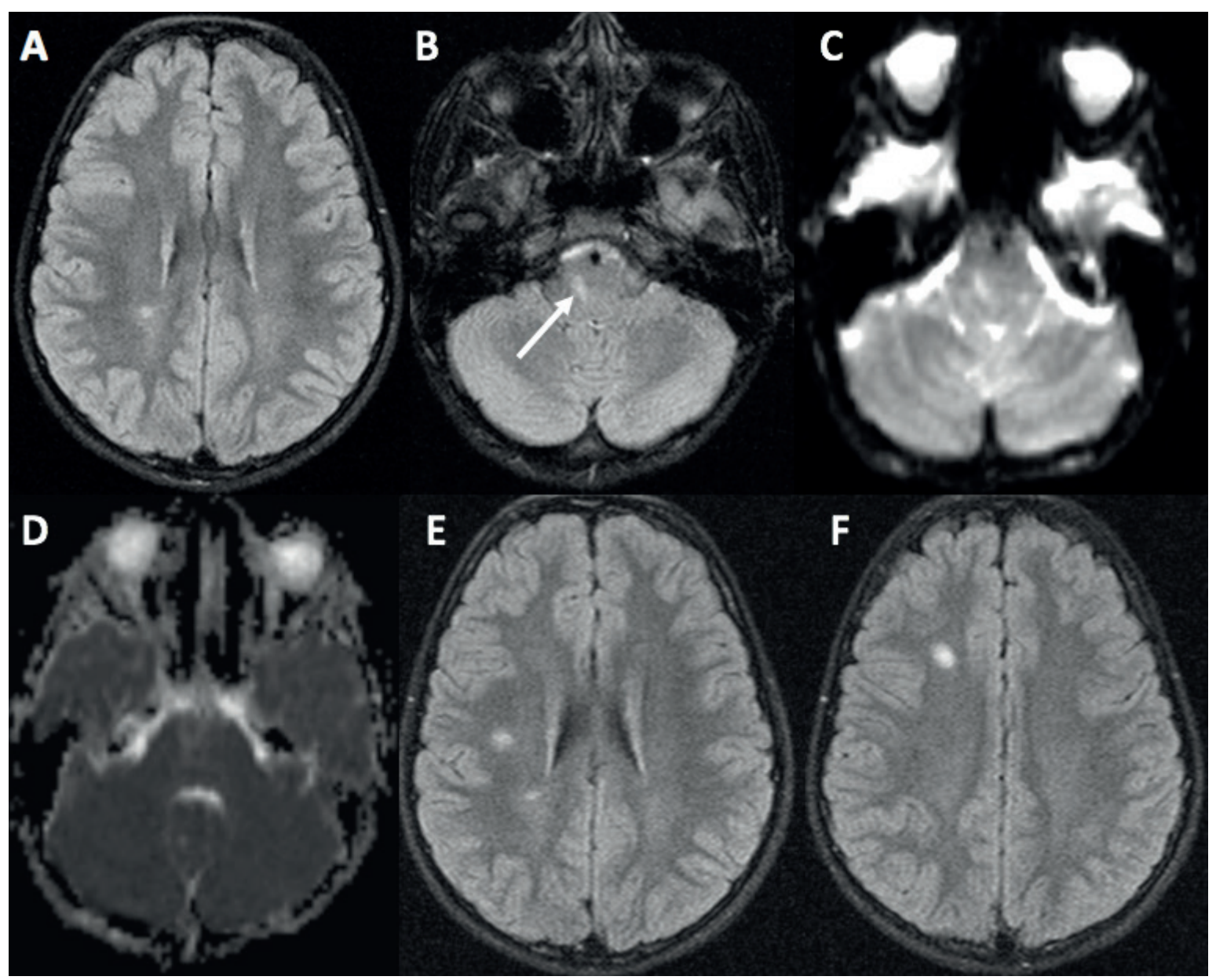

Fig. 1. The brain magnetic resonance imaging (MRI) of an 11-year-old boy presenting with diplopia who was diagnosed with MS 1 month after the first event. (A, B) Baseline axial FLAIR MRI of the brain revealing the hyperintense lesions in the periventricular white matter and brainstem (white arrow). (C, D) Diffusion-weighted imaging and ADC showing high signal intensity resembling stroke in the brain stem. (E, F) One month after the first event, FLAIR MRI showing new hyperintense lesions in the periventricular white matter. 
Table I. Demographic and clinical characteristics of patients.

\begin{tabular}{lccc}
\hline \multicolumn{1}{c}{$n$} & MS, at first event & CIS & $p$ \\
\hline Mean age at first event, years \pm SD (range) & $13.2 \pm 2.6(7.1-17)$ & $10.1 \pm 4.2(3-16.9)$ & 0.021 \\
Female/male, $n(\%)$ & $17 / 10(63 / 37)$ & $9 / 2(82 / 18)$ & 0.44 \\
Presentation & & & 0.06 \\
Optic neuritis, $n(\%)$ & $5(45)$ & $6(55)$ & \\
Brain stem / cerebellar, $n(\%)$ & $12(86)$ & $2(14)$ & \\
Spinal cord, n $(\%)$ & $1(50)$ & $1(50)$ & \\
Cerebral hemispheres, $n(\%)$ & $8(89)$ & $1(11)$ & \\
Multifocal, $n(\%)$ & $1(50)$ & $5 / 9(56)$ & 0.013 \\
CSF OCB positive, $n(\%)$ & $23 / 24(96)$ & & \\
\hline
\end{tabular}

$\mathrm{CSF}=$ cerebrospinal fluid, $\mathrm{CIS}=$ clinically isolated syndrome, $\mathrm{MS}=$ multiple sclerosis, $\mathrm{OCB}=$ oligoclonal band.

\section{Clinical features}

The age was younger in children with CIS than children with MS $(p=0.021)$. The age at first event was not statistically different between patients with different modes of presentation $(p=0.2)$. The mean age at first event was 11,3 \pm 3 years (range 6.7 to 14.7 years) in patients with optic neuritis. Female preponderance was present in both children with CIS and MS. The first event presented mostly in spring (34\%, $\mathrm{n}=13)$ and winter $(29 \%, \mathrm{n}=11)$ than in summer $(18 \%, n=7)$ and autumn $(18 \%, n=7)$. The season of the first event did not differ between patients with CIS and MS ( $p=0.72)$.

The modes of presentation were monofocal including brainstem/cerebellar syndrome $(n=14)$, optic neuritis $(n=11)$, dysfunction of cerebellar hemispheres $(\mathrm{n}=9)$, spinal cord syndrome $(\mathrm{n}=2)$ and multifocal $(\mathrm{n}=2)$. The ratio for developing MS among patients with brainstem/cerebellar syndrome, optic neuritis, dysfunction of cerebellar hemispheres, spinal cord syndrome, and multifocal involvement were respectively $86 \%, 45 \%, 89 \%, 50 \%$, and $50 \%$ during the follow-up period. Patients with dysfunction of cerebellar hemispheres and brainstem/cerebellar syndrome were more likely to develop clinically definite MS compared to patients with optic neuritis, spinal cord syndrome, and multifocal presentation. However, in terms of clinical presentation at onset, there was no statistically significant difference between patients with CIS and MS ( $p$ $>0.05$ ).

The oligoclonal band status was available for 33 patients. The CSF oligoclonal bands were positive in 23 children with MS $(96 \%, \mathrm{n}=25)$ and in 5 children with CIS $(56 \%, n=8)$. The children with brain lesions in the first brain MRI $(92 \%$, $\mathrm{n}=24 / 26$ ) were more likely to be positive for CSF oligoclonal bands than children without brain lesion $(57 \%, \mathrm{n}=4 / 7)(p=0.021)$. Children having CSF oligoclonal bands were more likely to have MS than CIS ( $p=0.013$ ) (Table I). The isoelectric focusing of CSF showed pattern I in 4 children with CIS and one with MS; pattern II in 4 children with CIS and 18 children with MS; pattern III in one with CIS and 5 children with MS. Isoelectric focusing pattern was significantly different between children with CIS and MS ( $p=0.026)$.

\section{Neuroimaging features}

Brain MRI of 38 patients and spinal cord MRI of 34 patients were available for assessment. Baseline MRI findings of the brain and spinal cord are shown in Table II. Significantly higher numbers of T2-hyperintense lesions were detected in patients with MS (mean $6.6 \pm 5.9$, range 0 to 26) compared to patients with CIS (mean $2.5 \pm 2.9$, range 0 to 8$)(p=0.015)$. The number of T1-hypointense lesions was not significantly different between children with MS (mean $1.3 \pm 3$, range 0 to 15 ) and children 
Table II. Baseline magnetic resonance imaging findings in patients with clinically isolated syndrome and multiple sclerosis.

\begin{tabular}{lccc}
\hline & $\begin{array}{c}\text { MS, at first } \\
\text { event }\end{array}$ & CIS & $\mathrm{p}$ \\
\hline \multicolumn{1}{c}{$n$} & 27 & 11 & \\
Number of T2-hyperintense lesions, mean (range) & $6.6(0-26)$ & $2.5(0-8)$ & 0.015 \\
Presence of periventricular lesion, $\mathrm{n}(\%)$ & $23(85)$ & $4(36)$ & 0.005 \\
Presence of juxtacortical lesion, $\mathrm{n}(\%)$ & $7(26)$ & $4(36)$ & 0.69 \\
Presence of infratentorial lesion, $\mathrm{n}(\%)$ & $16(60)$ & $2(18)$ & 0.03 \\
Presence of lesion of the corpus callosum, n (\%) & $12(44)$ & $0(0)$ & 0.008 \\
Presence of T1-hypointense lesion, n (\%) & $12(44)$ & $2(18)$ & 0.16 \\
Number of T1-hypointense lesions, mean (range) & $1.3(0-15)$ & $0.4(0-2)$ & 0.15 \\
Presence of gadolinium-enhancing lesion, n (\%) & $3(11)$ & $2(18)$ & 0.61 \\
Number of gadolinium-enhancing lesions, mean (range) & $0.3(0-4)$ & $0.5(0-4)$ & 0.54 \\
Presence of confluent lesion, n (\%) & $5(19)$ & $0(0)$ & 0.29 \\
Presence of giant lesion, $\mathrm{n}(\%)$ & $3(11)$ & $1(9)$ & 1.00 \\
& 26 & 8 & \\
Presence of spinal lesion, $\mathrm{n}(\%)$ & $7(27)$ & $2(33)$ & 1.00 \\
\hline
\end{tabular}

with CIS (mean $0.4 \pm 0.8$, range 0 to 2 ) ( $p=0.15$ ). The number of gadolinium-enhancing lesions did not differ between patients with MS (mean $0.3 \pm 0.9$, range 0 to 4 ) and children with CIS (mean $0.5 \pm 1.3$, range 0 to 4$)(p=0.54)$.

In the baseline MRI, the ratio of the presence of periventricular, infratentorial, corpus callosum, juxtacortical, spinal, and T1-hypointense lesions were $85 \%, 60 \%, 44 \%, 26 \%, 27 \%, 44 \%$ in children with MS and 36\%, 18\%, $0 \%, 36 \%, 33 \%$, $18 \%$ in children with CIS, respectively. The patients with periventricular, infratentorial, and corpus callosum lesions were more likely to have MS than CIS ( $p=0.005, p=0.03, p=0.008$, respectively). The presence of juxtacortical, spinal, T1-hypointense was not significantly different between patients with CIS and MS ( $p$ $>0.05$ ).

The presence of gadolinium-enhancing, confluent, and giant lesions were $11 \%, 19 \%$, $11 \%$ in children with MS and $18 \%, 0 \%, 9 \%$ in children with CIS, respectively. The presence of gadolinium-enhancing, confluent and giant lesions did not differ between patients with CIS and MS $(p>0.05)$.

\section{Discussion}

A total of 38 children with CIS aged 3-17 years were retrospectively evaluated. Age, sex, mode of clinical presentation, the status of the oligoclonal band in CSF, and baseline MRI findings of the brain and spinal cord were evaluated to determine their relevance to the development of MS.

Clinically definite MS developed in $71 \%$ of children after a mean follow-up of 11 months. The previous studies specified a risk from $15 \%$ to $62 \%$ for MS conversion during the follow-up duration of 2.2-7.6 years. ${ }^{8-13}$ The exclusion of children with CIS who had clinical and radiological features resembling other demyelinating diseases rather than MS (e. g. bilateral optic neuritis, longitudinally extensive transverse myelitis) may be responsible for a higher conversion rate to MS in our cohort.

In agreement with other studies of children with CIS, the older age at first demyelinating event and the presence of the oligoclonal band in the CSF tended to be more common in patients with MS than patients with CIS. ${ }^{14-17}$ It has been 
suggested that the change in cerebrospinal fluid components and hormonal profile with age were associated with an increased risk of progression to MS in older children. ${ }^{16,18}$ The CSF oligoclonal bands were more likely to be present in children with brain lesion in the first brain MRI than children without brain lesion ( $92 \%$ vs. $57 \%$ ). The presence of oligoclonal bands in the CSF which was found to be associated with a higher lesion load in the brain MRI has been suggested as an earlier finding of MS progression. ${ }^{19}$ Also, between children with CIS and MS, the isoelectric focusing pattern was different, which could be interpreted as either indicating different diseases or different stages of the same disease. Female sex was predominant in children with CIS and MS, in line with a previous study. ${ }^{20}$

The rate of conversion to MS differed according to the initial mode of clinical presentation. The brainstem/cerebellar or cerebral involvement was associated with the greatest risk of subsequent MS diagnosis. These clinical presentations were determined as the predictors of progression to MS in previous studies with long-term follow-up. ${ }^{8,21}$ The preponderance of brainstem dysfunction as the initial symptom of MS was also emphasized in children. ${ }^{21,22}$ The likelihood of MS was $45 \%$ in children presenting with optic neuritis, that is a greater ratio compared to previous studies (17-36\%). The mean age of children with optic neuritis was similar in our study to in previous studies (11.3 years vs. 10.2 to 12.2 years). However, the age ranges were broader in these studies, ranging from 2 to 17 years, than in our study (6.7 to 14.7 years). ${ }^{13,23-26}$ It was suggested that the risk of developing MS is correlated with age based on the differentiation of the immune system. ${ }^{27}$ This could elucidate why the ratio for the likelihood of MS was higher in our study than previous studies.

In terms of initial MRI findings, the number and localization of T2-hyperintense lesion were related with the development of MS. The increased number of T2-hyperintense lesion and the presence of the lesion in periventricular, infratentorial and corpus callosum were associated with a tendency for the development of MS, which is consistent with previous findings. ${ }^{14,16,20,28-30}$ The MRI lesion load, which has been suggested as an indicator of immune activation, were defined as a predictor for developing $\mathrm{MS}^{28}$ However, there was no difference observed between children with MS and CIS in the number of T1-hypointense lesions and gadolinium-enhancing lesions, and the presence of juxtacortical, spinal, T1hypointense, gadolinium-enhancing, confluent, and giant lesions.

The limitations of our study are the small sample size, relatively short-term follow-up period, and the absence of data regarding serum 25-hydroxyvitamin D status, EpsteinBarr-virus infection, and genotype which were suggested risk factors of progression to MS. ${ }^{20}$ The first event emerged mostly in spring and winter than in summer and autumn, that could be caused by the seasonal variation of the serum 25-hydroxyvitamin D status. However, we did not find a significant difference in the season of the first event between patients with CIS and MS. The data regarding antibodies against myelin oligodendrocyte glycoprotein was not available in our cohort. However, it has been suggested that these antibodies were absent in pediatric patients with relapsing-remitting MS. $^{31}$

In conclusion, we identified that older age at first event, the presence of CSF oligoclonal bands, a higher number of $\mathrm{T} 2$ lesions and periventricular, infratentorial and corpus callosum lesions were associated with a tendency for development of MS. These clinical and radiological features may be suggestive of development of MS in children with CIS.

\section{Acknowledgment}

This research did not receive any specific grant from funding agencies in the public, commercial, or not-for-profit sectors. 


\section{REFERENCES}

1. Banwell B, Ghezzi A, Bar-Or A, Mikaeloff Y, Tardieu M. Multiple sclerosis in children: Clinical diagnosis, therapeutic strategies, and future directions. Lancet Neurol 2007; 6: 887-902.

2. Chitnis T, Tenembaum S, Banwell B, et al; International Pediatric Multiple Sclerosis Study Group Consensus statement: Evaluation of new and existing therapeutics for pediatric multiple sclerosis. Mult Scler J 2012; 18: 116-127.

3. Miller DH, Chard DT, Ciccarelli O. Clinically isolated syndromes. Lancet Neurol 2012; 11: 157-169.

4. Tintore M, Rovira A, Rio J, et al. Defining high, medium and low impact prognostic factors for developing multiple sclerosis. Brain 2015; 138(Pt 7): 1863-1874.

5. Kappos L, Freedman MS, Polman CH, et al; BENEFIT Study Group Effect of early versus delayed interferon beta- $1 b$ treatment on disability after a first clinical event suggestive of multiple sclerosis: A 3-year follow-up analysis of the BENEFIT study. Lancet 2007; 370: 389-397.

6. Sadaka Y, Verhey LH, Shroff MM, et al; Canadian Pediatric Demyelinating Disease Network 2010 McDonald criteria for diagnosing pediatric multiple sclerosis. Ann Neurol 2012; 72: 211-223.

7. Krupp LB, Tardieu M, Amato MP, et al; International Pediatric Multiple Sclerosis Study Group International Pediatric Multiple Sclerosis Study Group criteria for pediatric multiple sclerosis and immune-mediated central nervous system demyelinating disorders: Revisions to the 2007 definitions. Mult Scler J 2013; 19: 1261-1267.

8. Peche SS, Alshekhlee A, Kelly J, Lenox J, Mar S. A long-term follow-up study using IPMSSG criteria in children with CNS demyelination. Pediatr Neurol 2013; 49: 329-334.

9. Krupp LB, Banwell B, Tenembaum S; International Pediatric MS Study Group Consensus definitions proposed for pediatric multiple sclerosis and related disorders. Neurology 2007; 68(16 Suppl 2): S7-S12.

10. Dale RC, Pillai SC. Early relapse risk after a first CNS inflammatory demyelination episode: Examining International consensus definitions. Dev Med Child Neurol 2007; 49: 887-893.

11. Neuteboom RF, Boon M, Catsman Berrevoets $\mathrm{CE}$, et al. Prognostic factors after a first attack of inflammatory CNS demyelination in children. Neurology 2008; 71: 967-973.
12. Alper G, Heyman R, Wang L. Multiple sclerosis and acute disseminated encephalomyelitis diagnosed in children after long-term follow-up: Comparison of presenting features. Dev Med Child Neurol 2009; 51 : 480-486.

13. Tantsis EM, Prelog K, Brilot F, Dale RC. Risk of multiple sclerosis after a first demyelinating syndrome in an Australian Paediatric cohort: Clinical, radiological features and application of the McDonald 2010 MRI criteria. Mult Scler J 2013; 19: 1749-1759.

14. Van der Vuurst de Vries RM, van Pelt ED, Mescheriakova JY, et al. Disease course after clinically isolated syndrome in children versus adults: A prospective cohort study. Eur J Neurol 2017; 24: 315-321.

15. Dale RC, Brilot F, Banwell B. Pediatric central nervous system inflammatory demyelination: Acute disseminated encephalomyelitis, clinically isolated syndromes, neuromyelitis optica, and multiple sclerosis. Curr Opin Neurol 2009; 22: 233-240.

16. Waldman AT, Stull LB, Galetta SL, Balcer LJ, Liu GT. Pediatric optic neuritis and risk of multiple sclerosis: Meta-analysis of observational studies. J AAPOS 2011; 15: 441-446.

17. Mikaeloff Y, Suissa S, Vallee L, et al; KIDMUS Study Group. First episode of acute CNS inflammatory demyelination in childhood: prognostic factors for multiple sclerosis and disability. J Pediatr 2004; 144 : 246-252.

18. Chabas D, Ness J, Belman A, et al; For the US Network of Pediatric MS Centers of Excellence Younger children with MS have a distinct CSF inflammatory profile at disease onset. Neurology 2010; 74: 399-405.

19. Tur C, Montalban X. CSF oligoclonal bands are important in the diagnosis of multiple sclerosis, unreasonably downplayed by the McDonald criteria 2010: No. Mult Scler J 2013; 19: 717-718.

20. Banwell B, Bar-Or A, Arnold DL, et al. Clinical, environmental, and genetic determinants of multiple sclerosis in children with acute demyelination: A Prospective National Cohort Study. Lancet Neurol 2011; 10: 436-445.

21. Ghezzi A, Pozzilli C, Liguori M, et al. Prospective study of multiple sclerosis with early onset. Mult Scler J 2002; 8: 115-118.

22. Ghezzi A, Deplano V, Faroni J, et al. Multiple sclerosis in childhood: Clinical features of 149 cases. Mult Scler J 1997; 3: 43-46. 
23. Wilejto M, Shroff M, Buncic JR, Kennedy J, Goia C, Banwell B. The clinical features, MRI findings, and outcome of optic neuritis in children. Neurology 2006; 67: 258-262.

24. Alper G, Wang L. Demyelinating optic neuritis in children. J Child Neurol 2009; 24: 45-48.

25. Heussinger N, Kontopantelis E, Rompel O, Paulides M, Trollmann R. Predicting multiple sclerosis following isolated optic neuritis in children. Eur J Neurol 2013; 20: 1292-1296.

26. Lucchinetti CF, Kiers L, O’Duffy A, et al. Risk factors for developing multiple sclerosis after childhood optic neuritis. Neurology 1997; 49: 1413-1418.

27. Chabas D, Ness J, Belman A; US Network of Pediatric MS Centers of Excellence Younger children with MS have a distinct CSF inflammatory profile at disease onset. Neurology 2010; 74: 399-405.
28. Kuhle J, Disanto G, Dobson R, et al. Conversion from clinically isolated syndrome to multiple sclerosis: A large multicenter study. Mult Scler J 2015; 21: 10131024.

29. Fisniku LK, Brex PA, Altmann DR, et al. Disability and T2 MRI lesions: A 20-year follow-up of patients with relapse onset of multiple sclerosis. Brain 2008; 131(Pt 3): 808-817.

30. Brex PA, Ciccarelli O, O'Riordan JI, Sailer M, Thompson AJ, Miller DH. A longitudinal study of abnormalities on MRI and disability from multiple sclerosis. N Engl J Med 2002; 346: 158-164.

31. Pröbstel AK, Rudolf G, Dornmair K, et al. AntiMOG antibodies are present in a subgroup of patients with a neuromyelitis optica phenotype. J Neuroinflammation 2015; 12: 46. 